\title{
EDITORIAL
}

\section{Hypoxia-driven mechanisms in lung biology and disease: a new review series of the ERS Lung Science Conference}

\author{
N. Weissmann
}

W ith the evolution of photosynthesis, organisms had to cope with a dramatic change in their environment: the presence of oxygen. Despite the initial toxicity of this gas, the presence of molecular oxygen offered a more effective means of energy metabolism. As a result, humans and animals require oxygen to survive and a shortage of oxygen, hypoxia, can evoke life-threatening conditions. To maintain an optimal oxygen level and thus, energy supply for the body, the lung is the first-line organ functioning as an interface between the body and the environment.

Both acute as well as chronic lung failure may lead to arterial hypoxaemia with subsequent cellular damage, organ failure and death. Hypoxia, however, not only affects the systemic circulation but also has widespread consequences for the lung itself.

Understanding the effects of hypoxia in the lung and the systemic circulation is thus pivotal to the development of new strategies to treat diseases associated with, or caused by, hypoxia.

The lung has developed a specialised architecture to optimise gas exchange and thus, the uptake of oxygen from the alveoli into the blood. A large surface and a thin diffusion barrier between the airspace and the blood are the structural prerequisites for efficient and rapid oxygen uptake [1]. But physiology is just as important. As the partial pressure of oxygen varies between different regions of the lung, even in healthy subjects but especially in patients with lung diseases, pulmonary blood flow and alveolar ventilation have to be matched. The regulatory process achieving this adaptation is known as hypoxic pulmonary vasoconstriction (HPV) or the von Euler-Liljestrand mechanism. A disturbance in HPV, as may occur in pneumonia, adult respiratory distress syndrome or liver failure, can cause life-threatening disturbances to oxygen uptake into the blood $[2,3]$. However, the molecular mechanisms of this important regulatory process are still not fully resolved [4]. Although recent advances in the field have identified key components of this pathway, we still do not

\section{STATEMENT OF INTEREST: None declared.}

CORRESPONDENCE: N. Weissmann, University of Giessen Lung Center, Medical Clinic $\| / N$, Klinkikstr. 36, 35392 Giessen, Germany. Fax: 49 6419942419. E-mail: Norbert.Weissmann@ innere.med.uni-giessen.de know the exact oxygen-sensing process underlying HPV. Reactive oxygen species (ROS) have been suggested to play an important role as mediators of this response. However, considerable controversy remains concerning the regulation of ROS generation in hypoxia, and future studies still have to address whether interference with ROS can be used therapeutically [5-7]. Recently, some advances have been made with regard to the involvement of membrane ion channels in the downstream mechanism of HPV $[8,9]$.

In generalised alveolar hypoxia, HPV becomes meaningless for the optimisation of blood flow but results in a general increase in pulmonary vascular resistance. If such conditions become chronic, in addition to the vasoconstrictor process, a vascular remodelling process causing thickening of the pre-capillary vascular wall occurs, resulting in pulmonary hypertension. Hypoxia-induced pulmonary hypertension not only occurs upon exposure to high altitude but can also be associated with a variety of pulmonary diseases, which are characterised by chronic alveolar hypoxia. Similar to the mechanisms of acute $\mathrm{HPV}$, the details of the molecular steps of this remodelling process, which range from oxygen sensing to cell proliferation, are not yet fully understood. A major advance has been the identification of the hypoxia-inducible transcription factor (HIF) family. The HIF family members are attractive targets for interference with the effects of hypoxia in the lung, not only with regard to hypoxia-induced pulmonary hypertension but also with regard to tumour growth, which involves HIFdependent target genes [10, 11]. However, detailed studies concerning the complex molecular biology of HIF regulation are still required to allow translation for use in the treatment of hypoxia-associated lung diseases.

Another possible consequence of alveolar hypoxia is the development of pulmonary oedema, a phenomenon known as high-altitude pulmonary oedema (HAPE). While the mechanisms leading to HAPE are yet to be resolved, the development of HAPE has been associated with disturbances in HPV and haemodynamics, inflammatory responses, and the effects of hypoxia on alveolar epithelial fluid transport. In the case of the latter, the effects of hypoxia are of major importance for alveolar fluid clearance in different types of alveolar oedema; for example, those associated with acute respiratory distress syndrome (ARDS) or pneumonia. Accordingly, a better understanding of the mechanisms regulating cellular 
adaptation to hypoxia may lead to the development of novel therapeutic strategies for treatment of ARDS and HAPE [12].

As well as the structural and physiological properties of the lung, the regulation of ventilation is a key feature to assure optimal oxygenation and avoid hypoxaemia. One aspect of disturbance to this system is the effect of nocturnal ventilatory abnormalities, which may lead to systemic hypertension. Again, our knowledge of the underlying pathways of this pathophysiological cascade is still limited [13].

A variety of questions emerge from these examples of the effects and consequences of hypoxia in the lung and the body, as follows.

How is oxygen sensed in acute and chronic hypoxia in the lung and body? What are the molecular mechanisms of HPV? What are the molecular steps in hypoxia-induced pulmonary vascular remodelling? How can interference with the hypoxiainducible factor be utilised to target lung diseases? What is the impact of hypoxia-dependent ROS-signalling on the acute and chronic vascular responses in the lung? What is the molecular link between hypoxia and pulmonary oedema? What links intermittent hypoxia to the development of systemic arterial hypertension? Answers to these question may provide a basis for the development of new strategies for the treatment of lung diseases, ranging from disturbances in gas exchange in the critically ill patient to, for example, the systemic effects of sleep apnoea and (lung) tumour growth.

Against this background, the European Respiratory Society covered the various aspects of hypoxia-driven mechanisms in the lung, from basic research to translational medicine, during their fourth and fifth Lung Science Conferences (which took place in 2006 and 2007, respectively). These Conferences took place in Taormina, Sicily, in an atmosphere that stimulated discussion between basic scientists, clinicians, experts in the field and young scientists. The translational approach of these Conferences facilitated the identification of those aspects of research that will prospectively have great impact on therapy of lung diseases in the future. To bring the key aspects of the Conferences to a larger audience, the European Respiratory Journal will present a new series on the different facets of the effects of hypoxia in humans, written by international experts in the field. The articles in this series will address recent advances with regard to the previously discussed key questions.

The series starts with an introduction to "The biology of oxygen" by WAGNER [14], followed by an article by ZHOU et al. [15] on the "Regulation of alveolar epithelial function by hypoxia". WAGNER [14] focuses on the complexity of the biology of oxygen, on oxygen transport and on the pathophysiology associated with a lack of oxygen, allowing easy access to this complex field of physiology and pathobiology. ZHOU et al. [15] then summarise current knowledge about the impact of hypoxia on alveolar epithelial cells, with an outlook on therapeutic implications in their detailed overview.

In future issues, the mechanisms of oxygen sensing, the molecular aspects of the regulation of hypoxia-inducible transcription, as well as sleep apnoea and hypoxic pulmonary vasoconstriction will be addressed in the series, which will provide a broad overview, spanning the multiple aspects of hypoxia in humans. The recent advances in the field of hypoxia-driven mechanisms in lung biology and disease will offer a variety of new options for the development of new strategies for the treatment of diverse lung diseases, thus helping us cope with the lack of oxygen.

\section{REFERENCES}

1 Weibel ER, Taylor CR. Functional design of the human lung for gas exchange. In: Fishman AP, ed. Pulmonary Diseases and Disorders. 3rd Edn. New York, McGraw-Hill, 1998; pp. 21-61.

2 Agusti AG, Rodriguez-Roisin R. Effect of pulmonary hypertension on gas exchange. Eur Respir J 1993; 6: 1371-1377.

3 Marshall BE, Hanson CW, Frasch F, Marshall C. Role of hypoxic pulmonary vasoconstriction in pulmonary gas exchange and blood flow distribution. 2. Pathophysiology. Intensive Care Med 1994; 20: 379-389.

4 Aaronson PI, Robertson TP, Knock GA, et al. Hypoxic pulmonary vasoconstriction: mechanisms and controversies. J Physiol 2006; 570: 53-58.

5 Waypa GB, Guzy R, Mungai PT, et al. Increases in mitochondrial reactive oxygen species trigger hypoxiainduced calcium responses in pulmonary artery smooth muscle cells. Circ Res 2006; 99: 970-978.

6 Weir EK, Archer SL. Counterpoint: hypoxic pulmonary vasoconstriction is not mediated by increased production of reactive oxygen species. J Appl Physiol 2006; 101: 995-998.

7 Weissmann N, Schermuly RT, Ghofrani HA, et al. Hypoxic pulmonary vasoconstriction - triggered by an increase in reactive oxygen species? Novartis Found Symp 2006; 272: 196-208.

8 Archer SL, London B, Hampl V, et al. Impairment of hypoxic pulmonary vasoconstriction in mice lacking the voltage-gated potassium channel Kv1.5. FASEB J 2001; 15: 1801-1803.

9 Weissmann N, Dietrich A, Fuchs B, et al. Classical transient receptor potential channel 6 (TRPC6) is essential for hypoxic pulmonary vasoconstriction and alveolar gas exchange. Proc Natl Acad Sci USA 2006; 103: 19093-19098.

10 Maxwell PH. The HIF pathway in cancer. Semin Cell Dev Biol 2005; 16: 523-530.

11 Semenza GL. Involvement of hypoxia-inducible factor 1 in pulmonary pathophysiology. Chest 2005; 128: Suppl. 6, 592S-594S.

12 Maggiorini M. High altitude-induced pulmonary oedema. Cardiovasc Res 2006; 72: 41-50.

13 Schulz R. The vascular micromilieu in obstructive sleep apnoea. Eur Respir J 2005; 25: 780-782.

14 Wagner PD. The biology of oxygen. Eur Respir J 2008; 31: 887-890.

15 Zhou G, Dada LA, Sznajder JI. Regulation of alveolar epithelial function by hypoxia. Eur Respir J 2008; (In press). 